\title{
Warum Vorschulkinder nicht zurückgrïssen müssen. Beobachtungen zu Spezifika des kindlichen Sequenzmusters in der Grusshandlung
}

\author{
Katrin Schaad/Esther Zollinger (Zürich)
}

\begin{abstract}
The pilot study takes into focus a so far widely unexamined branch of language acquisition and developmental psychology. The object of investigation is the salutatory behavior of children between four and seven years of age. The analysis of data gathered in a Swiss kindergarten allows deriving a pattern of sequences for the preschooler's salutatory habits. Children tend to remain passive during greeting episodes or perform fragmentary salutations: They follow their own rituals of greeting.

The childish salutatory behavior differs from the conventionalized norms valid for adults. This divergence, which is often considered deficient and reduced to a lack of politeness on the child's part, is in fact a normal state in the process of socialization A notable difference between the four- and the seven-year-olds has been observed: The older the children are, the more do their greetings correspond to the salutatory rituals conventionalized in society.

This allows the conclusion that greeting behavior is not acquired simultaneously with language - preschoolers have already acquired language to a large extent, while they are still in midst of the process of socialization.
\end{abstract}

\section{$1 \quad$ Einleitung}

Kinder im Vorschulalter sind in der Beherrschung der grammatischen Grundstrukturen mit Erwachsenen vergleichbar. Dieser Teil der sprachlichen Entwicklung ist bei Kindergartenkindern mehrheitlich abgeschlossen (cf. Oksaar 1987: 11). Sie können bereits eine infinite Anzahl Sätze generieren, auch wenn ihnen noch nicht das ausdifferenzierte Lexikon eines Erwachsenen zur Verfügung steht. Der eingeschränkte Umfang des Wortschatzes ist aber nicht die einzige Komponente, welche den Sprachgebrauch von Kindern von demjenigen der Erwachsenen unterscheidet. Zusätzlich zum lexikalischen und grammatikalischen Wissen müssen die Kinder Wissen über die Verwendung der Sprache, also Sprachhandlungswissen, erwerben. Dieses beinhaltet das Beherrschen unzähliger Konventionen und ist der Schlüssel zur gesellschaftlichen Integration.

Durch den Spracherwerb wird das Kind zu einem

Teil einer bestimmten sozialen Gemeinschaft und damit erst ein Mensch. Mit der Sprache lernt es Gefühle, Wünsche, Gedanken in sozial festgelegter Weise auszudrücken. Es lernt, dass man nicht immer reden darf, zu wem man will und wann man will; es lernt, dass man manchmal reden muss, obwohl man nicht will [...]; es lernt über die Sprache all jene kulturellen, moralischen, religiösen und sonstigen Vorstellungen der jeweiligen Gemeinschaft kennen, die diese über eine Gemeinschaft von Ameisen hinausheben (Klein 1989: 10). 
Diese "präskriptiven und proskriptiven Normen" (Oksaar 1987: 107), welche die Sprachhandlungskonventionen festlegen, bestimmen auch das Verwenden von Grüssen. Der Gruss ist also eine sprachliche Routinehandlung und Teil der kommunikativen Kompetenz. Erst und einzig durch die Ausführung in der Kommunikationssituation erhält er Bedeutung als Bekundung von sozialer Anerkennung. Im Unterschied zu anderen Routinehandlungen wie Bitten und Danken variieren die einzelnen Ausführungen der Grusshandlung stark, da eine grosse Auswahl an Floskeln und Gesten zur Selektion und Kombination zur Verfügung steht. Je nach sozialer Konstellation und Situationsdefinition setzt sich die Grusshandlung anders zusammen, was sowohl auf verbaler wie auf nonverbaler Ebene sichtbar ist und den Gruss zu einem komplexen Phänomen macht.

In the manner in which the greeting ritual is performed, the greeters signal to each other their respect of social status, their degree of familiarity, their degree of liking for one another, and also, very often, what roles they will play in the encounter that is about to begin (Kendon 1990: 154).

Es bedarf eines fundierten sozialen und kulturellen Wissens, um jeweils gegenüber der richtigen Person im richtigen Moment den angemessenen Gruss zu äussern. Denn wie alle Routinehandlungen, deren Beherrschung die kommunikative Kompetenz ausmacht, ist auch die Grusshandlung klar strukturiert.

Für Erwachsene folgt auf einen Gruss genauso selbstverständlich ein Gegengruss wie auf eine Frage eine Antwort. Die beiden Elemente stehen jeweils in konditioneller Relevanz zueinander, was bedeutet, dass gewöhnlich auf das erste das zweite folgt (cf. Schegloff 1972: 364). Wenn die erwartete Reaktion auf das erste Element ausbleibt, hat dies Irritation zur Folge; man behilft sich mit einer konversationellen Implikatur. Da die Zusammengehörigkeit der beiden Elemente konventionell festgesetzt ist und im Normalfall eingehalten wird, lassen sich Sequenzmuster für die Interaktion ableiten. Solche Muster können für Grusshandlungen ebenso erstellt werden wie für andere verbale und nonverbale soziale Routinehandlungen.

Die vorliegende Pilotstudie untersucht das Grussverhalten von Kindern zwischen vier und sieben Jahren. Der Fokus liegt dabei auf dem Ablauf der Grusshandlungen, woraus ein Sequenzmuster des kindlichen Grusses abgeleitet und interpretiert wird. Die Datenerhebung, die dieser Arbeit zugrunde liegt, ${ }^{1}$ lässt keine abschliessenden Aussagen über das Grussverhalten von Kindern zu, da das Material von zu geringem Umfang ist. Jedoch können allgemeine Tendenzen über das kindliche Sequenzmuster in Grussakten erkannt und Eigenheiten, die aus den Besonderheiten des untersuchten Kindergartens hervorgehen, festgestellt werden.

\section{These}

Kinder halten die für Erwachsene konventionalisierten Sequenzmuster in den Grussritualen nur bedingt ein. In der Kommunikation unter Vier- bis Siebenjährigen fehlt der Gruss häufig, oder aber seine Ausführung folgt nicht dem Sequenzmuster, welches sich unter Erwachsenen etabliert hat. Auch im Gruss-"Austausch" mit Erwachsenen weichen Kinder oft von diesem ab. Dies lässt darauf schliessen, dass der Sozialisationsprozess, "in dem das Kind lernt, die Sprache in einer [den Standards der Erwachsenen] sozial angemessenen Weise zu verwenden" (Oksaar 1987: 107), noch nicht abgeschlossen ist. Die für Erwachsene gebräuchlichen Sequenzmuster der Grusshandlung sind dementsprechend (noch) nicht mit dem Spracherwerb $^{2}$ verinnerlicht worden.

\footnotetext{
${ }^{1}$ Cf. Kapitel 3.

2 Mit "Spracherwerb" ist im Weiteren die von Oksaar als "sprachliche Ontogenese" (1987: 12) bezeichnete Phase gemeint (cf. Kapitel 2).
} 


\section{$3 \quad$ Grussbegriff und Konzepte zur Grusshandlung}

Das Grussverhalten von Kindern im Vorschulalter wurde in der Forschung bisher nur am Rande untersucht. Deshalb stützt sich die vorliegende Studie sowohl auf linguistische Arbeiten zur Interaktion, insbesondere zum Thema Grüssen, als auch auf Erkenntnisse der Sprachentwicklungspsychologie. Die zwei Konzepte 'Sequenz' und 'Adjacency Pair' aus der Gesprächslinguistik sind dabei von besonderer Bedeutung. Die Sequenz ist eine "wahrnehmbare Folge von Einheiten einer Äusserung [...] mit Abhängigkeitsbeziehungen zwischen den einander berührenden Einheiten" (Abraham 1988: 752). Das Paradebeispiel einer solchen Abfolge von sprachlichen Handlungen ist die Paarsequenz, das 'Adjacency Pair'. Wie im Beispiel 'Frage und Antwort' hat in der Paarsequenz das erste Element "konditionale Relevanz für das zweite" (Glück 2005: 588), oder anders ausgedrückt, "given the first, the second is expectable" (Schegloff 1972: 364). Der Begriff Sequenz wird allerdings nicht bloss für die Einheiten einer Äusserung verwendet, sondern für die ganze Grusshandlung, was auch deren nonverbale Elemente einschliesst.

In der vorliegenden Untersuchung wird der weitgefasste Grussbegriff Kendons übernommen:

The term 'greeting' will be used to refer to that unit of social interaction often observed when people come into one another's presence, which includes a distinctive exchange of gestures or utterances in which each person appears to signal to the other, directly and explicitly, that he has been seen" (Kendon 1990: 153).

Kendon sequenziert in seiner Theorie den Gruss in die Phasen 'pre-interactional steps' 'distance salutation', 'approach phase', 'final approach', 'close salutation' und 'end of salutation' (cf. Kendon 1990). Jeder dieser Phasen ordnet er spezifische Elemente der Grusshandlung, sogenannte "behavioral units" (Kendon 1990: 159), zu und entwickelt damit eine detaillierte, für die Verhaltensforschung bedeutsame Sequenznorm menschlicher Grüsse. Dabei steht nicht eine Interpretation der beobachteten Grusspraktiken im Vordergrund, sondern "a description of how people greet one another" (Kendon 1990: 158). Die für die vorliegende Arbeit bedeutendsten 'behavioral units' "body cross"3 und "grooming"4 (Kendon 1990: 185) werden der 'approach phase' zugeordnet.

Dem Salutations-Schema Kendons folgend, wird in der vorliegenden Studie das nonverbale Verhalten im Grussakt in die Betrachtung miteinbezogen. Dabei wird Kendons Sequenzmuster für Grüsse als Standard für den Ablauf der Grusshandlung unter Erwachsenen angesehen. Wenn also im Weiteren vom "üblichen Sequenzmuster" die Rede ist, so bezieht sich dies auf das unter Erwachsenen normale Grussverhalten. ${ }^{5}$ Die Anwendung dieses integrierenden Ansatzes auf das kindliche Grussverhalten erlaubt es, dieses ganzheitlich zu erfassen; der Gruss wird somit als kommunikative Praktik verstanden. Allerdings kommt dem sprachlichen Teil der Begrüssung in der vorliegenden Analyse eine grössere Bedeutung zu als in Kendons verhaltensorientierter Untersuchung. Wichtig hierfür ist Goffmans Studie Das Individuum im öffentlichen Austausch (1982), welche, im Kontext interpersoneller Rituale, Grusshandlungen als "rituelle Klammern für eine Vielfalt von gemeinsamen Aktivitäten"

\footnotetext{
3 "It [body cross] may take a variety of forms. Either one arm may be moved across the mid-line of the body, or, in some examples, both arms are brought into play, the hands being clasped together in front. In other instances an object the individual is carrying may be raised up in front. In still other instances, which we have counted as examples, the hand is raised to the mouth or it is raised to grasp the neck" (Kendon 1990: 185).

4 "We counted as groom any instances where $p$ adjusts his clothing - straightening a sweater or a tie, for example; where he stokes or smooths his hair; scratches his head; pats himself or appears to brush something of himself, or rubs any part of his face" (Kendon 1990: 186).

5 Die hier etablierte Norm bezieht sich auf Schweizerdeutsch sprechende Erwachsene mit guter Bildung aus der oberen Mittelschicht.
} 
(Goffman 1982: 118) betrachtet. Goffman definiert den Gruss als Zugänglichkeitsritual, welches als rituelle Kundgabe einen "Wechsel des Zugänglichkeitsgrads" (Goffman 1982: 119) markiert. Die Wahl einer bestimmten Grussformel wird nach Goffman durch den Zugänglichkeitsgrad, die Enge der Beziehung und das rituell etablierte Verhältnis der am Gruss beteiligten Personen beeinflusst. Diese Faktoren finden deshalb auch in der Betrachtung des kindlichen Grussverhaltens Berücksichtigung.

Kohrt (1985) bezeichnet den "Austausch bestimmter Routineformeln sowohl am Beginn als auch am Ende kommunikativer Interaktionen" (Kohrt 1985: 174) als Grüsse und geht darin mit Goffman überein. Im Folgenden wird dieser Grussbegriff, welcher sowohl Begrüssungen als auch Verabschiedungen umfasst, übernommen. Ebenso werden nachgestellte Befindlichkeitsfragen zum verbalen Teil der Grusshandlung gezählt, während Kohrt den Grussbegriff sehr eng hält und auf Floskeln, welche als Echogrüsse ausgetauscht werden, reduziert.

Zur Beantwortung der Frage, inwiefern Grusshandlungen erlernt werden müssen, werden die Arbeiten von Klein (1989) und Oksaar (1987) zur Sprachentwicklung herbeigezogen. Klein betont die Wichtigkeit des Spracherwerbs für die Sozialisation. Oksaar teilt diesen in verschiedene Stadien:

Unter sprachlicher Ontogenese verstehe ich die Entwicklung der Sprache in der Periode des Baby- und Kleinkindalters. Da die Entwicklung alle Komponenten des Sprachsystems umfasst von den ersten Phonemisierungs- und Symbolisierungsprozessen bis zur Bildung der grösseren sprachlichen Einheiten -, können wir, ohne allzu scharfe Grenzen zu ziehen, die Entwicklung der sprachlichen Verhaltensweisen der ersten 4 bis 5 Jahre hierher rechnen (Oksaar 1987: 12).

Dies lässt eine Verortung des Erlernens der Grusspraktik innerhalb der Sprachentwicklung zu.

\section{$4 \quad$ Korpus}

\subsection{Datenquelle und Probanden}

Die in unserer Arbeit untersuchten Begrüssungen und Verabschiedungen stammen aus Videoaufnahmen von insgesamt rund 80 Minuten Spielzeit. Diese wurden im Mai 2007 in einem privaten Montessori-Kindergarten am Zürichberg, am ersten Schultag nach den Frühlingsferien ${ }^{6}$, erstellt. ${ }^{7}$ Die 20 an der Datenerhebung beteiligten Kinder waren zum Zeitpunkt der Erhebung zwischen vier und sieben Jahre alt ${ }^{8}$ und besuchten diesen Kindergarten seit mindestens neun Monaten, jeweils vier bis zehn Halbtage pro Woche.

\subsection{Der untersuchte Kindergarten}

Da es sich um einen privaten Kindergarten handelte, stammten die Kinder überwiegend aus gutsituierten Familien aus der ganzen Stadt Zürich. Die acht Jungen und zwölf Mädchen waren vornehmlich schweizerdeutscher oder deutscher Muttersprache, warum im Kindergarten auch viel Hochdeutsch gesprochen wird. Der Unterricht wurde in schweizerdeutscher Sprache abgehalten. Der Leitsatz der Montessori-Pädagogik lautet "Hilf mir, es selbst zu tun" (Montessori 1983: Vorwort), was den individuellen Entwicklungsphasen des einzelnen Kindes spezielle Bedeutung zukommen lässt. Dieses Erziehungsmodell bedingt besondere Betreuungsverhältnisse; im betreffenden Kindergarten wurden die 20 Kinder von einer ausgebildeten Kindergärtnerin und drei Praktikantinnen betreut, mit denen sie per Du waren.

\footnotetext{
${ }^{6}$ Es wurde erwatet, dass Grusssequenzen nach den Ferien besonders intensiv und daher gut beobachtbar seien.

${ }^{7}$ Die Eltern wurden drei Wochen im Voraus schriftlich über die Datenerhebung informiert.

8 Die Untersuchung dieser Altersklassen scheint besonders interessant, da die Phase der "sprachlichen Ontogenese" (cf. Oksaar 1987: 12), welche bei den jüngeren Kindern noch voll im Gang ist, bei den älteren schon abgeschlossen ist.
} 
Ein weiteres Grundprinzip der Montessori-Pädagogik ist die Idee der jahrgangsübergreifenden Lerngruppen. Diese hat zum Ziel, dass die jüngeren Kinder von den älteren lernen und die älteren sich zugleich im Anleiten üben können. Die Kinder im untersuchten Kindergarten waren zwischen 2000 und 2004 geboren; somit waren Vier- bis Siebenjährige Teil derselben Lerngruppe. Der Unterricht im Montessori-Kindergarten soll die "Möglichkeit zur Selbsttätigkeit, freier Arbeitswahl und individuellem Lerntempo" (Montessori 1983: Vorwort) bieten. "Material für Übungen des täglichen Lebens und das Sinnesmaterial ermöglichen früh Erziehung und Entwicklung des Geistes. Selbsttätigkeit führt zur Selbständigkeit" (ebd.). Die dreistündigen Unterrichtsblöcke wurden durch geleitete Lektionen umrahmt, welche im Kreis ${ }^{9}$ stattfanden.

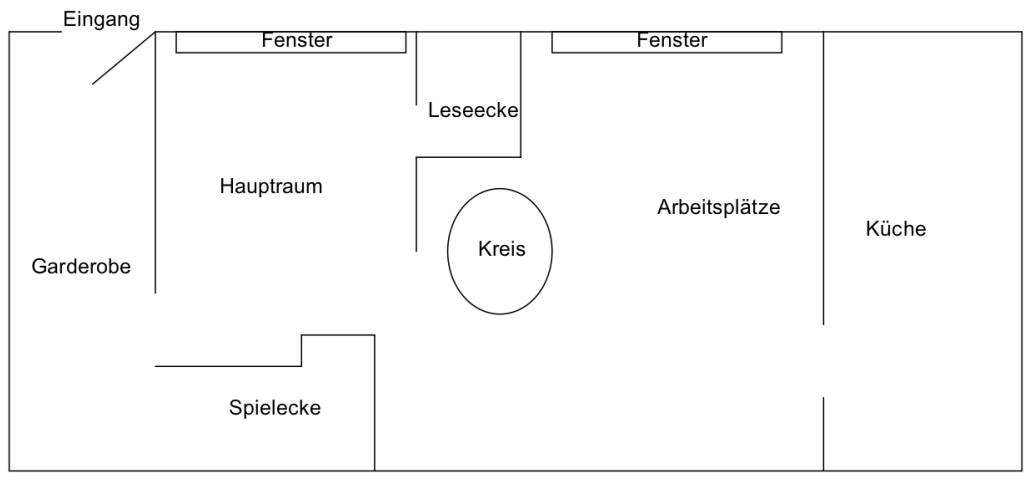

Abbildung 1: Grundriss des untersuchten Kindergartens

\subsection{Datenmaterial}

Die Hauptquelle der erfassten Daten war die erste Videosequenz von ca. 40 Minuten Spielzeit, welche einige Minuten lang im Hauptraum und danach in der Garderobe des Kindergartens während der Auffangzeit ${ }^{10}$ am Morgen aufgenommen wurde. Der Film zeigt die in dieser Zeit erfolgten Begrüssungen und Verabschiedungen. Die Begrüssungen fanden sowohl unter den Kindern wie auch zwischen Kindern und Betreuerinnen statt, während die Verabschiedungen ausschliesslich zwischen Kindern und Eltern stattfanden. Aus dem Datenmaterial wurden 34 Grusssituationen zur genaueren Analyse verwendet. 19 davon waren Begrüssungen zwischen Erwachsenen (von denen alle, mit Ausnahme eines Vaters, weiblich waren) und Kindern, acht waren Begrüssungen unter Kindern und sieben waren Verabschiedungen zwischen Eltern und Kindern.

Die zweite Videosequenz von ebenfalls rund 40 Minuten zeigt eine Lektion, die von den Untersuchungsleiterinnen mit den Kindern zum Thema "Gruss und Abschied" gestaltet wurden. Die durchgeführte Gesprächsrunde und ein Rollenspiel waren nicht direkt Untersuchungsgegenstände, lieferten aber ergänzende Gedanken.

\section{Zur Untersuchungsmethode: Transkription, Kategorisierung und Analyse der Grusssituationen}

Nach der Datenerhebung wurden die aufgezeichneten Grusssituationen transkribiert und einzeln analysiert. Das Datenmaterial wurde in drei Kategorien eingeteilt, welche die Konstellation der am Gruss beteiligten Personen berücksichtigen. So ergaben sich die Kategorien 'Begrüssungen zwischen Erwachsenen und Kindern', 'Begrüssungen unter Kindern' und 'Verabschiedungen zwischen Erwachsenen und Kindern'. Jede Grussszene wurde detailliert

\footnotetext{
${ }^{9}$ Der "Kreis" bildet als Versammlungsort das Herzstück des Montessori-Kindergartens; cf. Montessori (1983).

${ }^{10}$ Mit "Auffangzeit" ist die flexible Ankunftszeit vor Unterrichtsbeginn gemeint, in der die Kinder selbständig arbeiten.
} 
betrachtet und nach einem Kriterienkatalog beurteilt. Dies ermöglichte einen Vergleich, der allfällige Tendenzen bezüglich des Sequenzmusters im kindlichen Grussverhalten sichtbar machte. Anschliessend wurden die Ergebnisse mit entwicklungspsychologischen Erkenntnissen in Verbindung gebracht.

\subsection{Transkription und Analyse}

Die Transkription des Verbalen und Paraverbalen erfolgte nach dem gesprächsanalytischen Transkriptionssystem GAT (Deppermann 1999) und den Richtlinien des Deutschen Seminars für die Transkription des Schweizerdeutschen (Burger et al. 1998). Um die Kommunikationssituation darzustellen, wurde der Transkription ein kurzer Situationsbeschrieb vorangestellt, in dem die Kinder mit ihrem Jahrgang aufgeführt sind. Dem schweizerdeutschen Transkript wurde interlinear eine hochdeutsche Übersetzung beigefügt. Das nonverbale Grussverhalten wurde ausschliesslich bei den Kindern betrachtet und eine Beschreibung desselben wurde an die Transkription angefügt. Eine Transkriptionspassage kann mehrere Grusssituationen enthalten, welche sich teilweise überschneiden.

Um die Transkription der Grussszenen zu illustrieren, sei hier eine solche aufgeführt. Die Namen der beteiligten Personen wurden geändert.

Sequenz Nr. 8

Die Türe zum Kindergarten ist geschlossen. Frederik (2002, 5-jährig), seine Schwester Elisabeth (2005, 2-jährig) und die Mutter der beiden stehen in der Garderobe. Linus (2000, 7-jährig) kommt eilig aus dem Hauptraum, gefolgt von Fabio (2001, 6-jährig). Er öffnet die Tür und tritt der ankommenden Tanja (Kindergärtnerin) entgegen. Später tritt Lorena (2001, 6-jährig) ebenfalls an die Tür. Danach kommen Julian (2001, 6-jährig) und seine Mutter von aussen auf den Eingang zu und bleiben vor Tanja, die in der Türe Halt gemacht hat, stehen.

01 Tanja ho:i LInus HO:i; (.) schöni FErie kha- (-) hoi-

Hallo Linus, Hallo. Schöne Ferien gehabt? Hallo...

02 Linus =und ICH hab heut geBURTStag-

und ich habe heute Gebutstag.

03 Tanja [fabio] $(-)<<$ h $>$ grad hüt- (.) OH ich gratuLIER der he:; $>$ heieiei;

... Fabio. Gerade heute? Oh, ich gratuliere dir, he. Heieiei.

04 Linus ich bin SIEben geWORden;

Ich bin sieben geworden.

05 Tanja hoi LOr'- ja jetzt WEISS ich !MENNSCH! jetzt wird s ZIIT für d SCHUEL (.) hä?

Hallo Lor - ja jetzt weiss ich.. Mensch. Jetzt wird es Zeit für die Schule, hä?

Lorena am ACHTtundzwanzigsten apRIL hab ich geBURTStag geHABT;

Am 28. April habe ich Geburtstag gehabt.

07 Tanja öh du HÄSCH (.) blblbl dänn MÜEM mer ja NUR no FIIRE jetzt im CHINDsgi; (-) hä ich gratuLIER der $\ddot{\mathrm{A}}$ zum NACHhinein.

Öh. du hast. Blblbl, dann müssen wir ja nur noch feiern jetzt im Kindergarten. Hä? Ich gratuliere dir auch noch im Nachhinein.

08 Linus [ICH hab noch] KUchen mitgeBRACHT-

Ich hab noch Kuchen mitgebracht.

09 Tanja ou SUper dänn git s ja hüt GRAD en SCHÖne ZMORGE; (--) HOoi; JUlianc hoi hoic (--) hä

Oh super, dann gibt es ja heute ein schönes Frühstück. Hallo Julian, hallo, hallo

10 Mutter seisch Ä no HOI de TANja-

Sagst du auch noch hallo der Tanja. 
Tanja [guet] CHÖmed Inec (2.0) ja !HOoi! zäme hoi FREderikc häsch schöni FErie kha; (--) JA? (2.0) er STRAALT uf JEde FALL; (.) eLIsabeth ho:ic (-) ((lacht)) (2.0) GUEte MO:rge mitenAND-

Gut, kommt herein. Ja hallo zusammen. Hallo Frederik, hast du schöne Ferien gehabt? Ja? Er strahlt jedenfalls. Elisabeth, hallo. Guten Morgen miteinander.

\section{Nonverbales Verhalten:}

Linus umarmt Tanja während Turn 01. Bei Turn 03 lösen sie die Umarmung auf und Linus beginnt, mit Tanjas Foulard zu spielen. Fabio drängt sich zwischen die beiden. Dann nimmt Tanja Linus' Hände, lässt sie wieder los und streicht ihm durch die Haare (Turn 05). Bei Turn 06 nimmt Lorena Tanjas Hand und lässt sie während Turn 07 wieder los. Linus zieht Tanja während Turn 08 das Foulard aus, worauf sie es ihm aus der Hand nehmen will; er lässt es aber erst bei Turn 11 los. Julians Mutter winkt Tanja zu, lehnt sich nach Turn 09 zurück und spricht Julian in dieser Position an (Turn 10). Während Turn 11, nachdem Lorena und Linus zurück in den Hauptraum gegangen sind, beugt sich Tanja zu Frederik, seiner Schwester und der kauernden Mutter hinunter. Bis zum "JA" schaut sie Frederik direkt an und sucht dann Blickkontakt zur Mutter, die den Kopf schüttelt. Von Frederik ist keine verbale Reaktion hörbar. Tanja steht auf und kneift im Vorbeigehen Elisabeth in den Bauch. Dann richtet sie sich vollends auf, geht auf den Durchgang zu und tritt mit den letzten Worten in den Hauptraum.

Auf die Transkription der einzelnen Grussszenen in der Untersuchung folgte eine Feinanalyse, in der Auffälligkeiten festgehalten und das Grussverhalten der jeweiligen Personen in der konkreten Situation gedeutet wurde.

\section{Die Feinanalyse der oben transkribierten Grussszene ist unten exemplarisch abgebildet.}

\section{Sequenz Nr. 8}

Einzig Tanja wird von den Kindern bei ihrer Ankunft am Eingang erwartet und mehrfach umarmt. Daraus ist ersichtlich, dass Tanja als die Leiterin des Kindergartens und älteste Betreuungsperson eine besondere Stellung einnimmt und ein anderes Verhältnis zu den Kindern hat als die Praktikantinnen. Diese werden von den Kindern, wenn überhaupt, weniger überschwänglich und mit weniger sprachlichem und körperlichem Aufwand begrüsst. Die Beziehung zwischen den am Gruss beteiligten Personen spielt für die Ausgestaltung desselben eine wichtige Rolle: "Auf jeden Fall gilt, dass für Begrüssungs- und Abschiedsrituale nicht nur der Aspekt der Zugänglichkeit, sondern auch der des rituell etablierten Verhältnisses zwischen den Ausführenden von entscheidender Bedeutung ist" (Goffman 1982: 123). Der grosse Aufwand, den die Kinder im Grussakt betreiben, ist aber nur nonverbal. Keines der drei Kinder, die zur Begrüssung mit Tanja Körperkontakt initiieren, grüssen sie verbal zurück. Obwohl Tanja jedes Kind einzeln und mit Namen begrüsst, unterlassen diese den bei Erwachsenen üblichen verbalen Gegengruss und beginnen sogleich zu erzählen. Linus reagiert verbal weder auf die wiederholte Grussfloskel noch auf die an ihn gerichtete Befindlichkeitsfrage, wodurch er das Sequenzmuster, mit welchem Erwachsene üblicherweise beidseitig die Phase erhöhter Zugänglichkeit einleiten, nicht befolgt. Während bei Linus mit der Umarmung ein Element des Grussaktes vorhanden ist, unterlässt Lorena alles, was in Begrüssungen unter Erwachsenen als zur Grusshandlung zugehörig gesehen wird. Dieses Verhalten könnte als Hinweis dafür gesehen werden, dass im kindlichen Sequenzmuster die Phase erhöhter Zugänglichkeit nicht unbedingt beidseitig eingeleitet werden muss. Es scheint auszureichen, wenn die Kinder sich dadurch, dass sie begrüsst werden, der Aufmerksamkeit ihres Gegenübers sicher sind. Durch seine Erzählung unterbricht Linus Tanjas Begrüssung der umstehenden Kinder. Damit hält er sich nicht an das Muster des Turn-Taking, welches einer üblichen Interaktion zugrunde liegt. ${ }^{1}$ Auch nimmt er keine Rücksicht auf die von Lorena initiierte Konversation mit Tanja, indem er wiederum unaufgefordert erzählt, dass er Kuchen mitgebracht habe.

Auffällig ist weiter das hohe Tonregister Tanjas, welches als paraverbales Element typisch für die "nursery language" (vgl. Oksaar 1987: 122) ist. Zu dieser Sprachvariante gehören ebenso inhaltslose Ausdrücke wie "heieiei" und "blblbl". Im Gegensatz zu den Kindern ist der sprachliche Aufwand, den Tanja zur Begrüssung betreibt, auffällig hoch, was ihre erhöhte Zugänglichkeit den Kindern gegenüber anzeigt. Der Eindruck, dass diese besonders den Kindern gilt, wird verstärkt durch die Tatsache, dass Tanja Frederiks am Boden kauernde Mutter nicht explizit begrüsst, als sie an dieser vorbeigeht, sondern vor allem den Kindern Aufmerksamkeit widmet. Auch Julian wird deutlich von Tanja begrüsst, was dieser nicht erwidert. Darauf fordert ihn seine Mutter auf, zurückzugrüssen. Dies zeigt, dass die Eltern den Kindern das Grüssen, was diese nicht immer von sich aus tun, bewusst beibringen wollen. Dieses elterliche Verhalten wird auch von Blank/Berko beschrieben: "While

${ }^{11}$ Auch in der Interaktion zwischen Erwachsenen kann unter bestimmten Umständen vom Muster des TurnTakings abgesehen werden, so zum Beispiel bei einer dringenden Mitteilung eines Kommunikationspartners an den andern. Linus scheint es für wichtig zu halten, Tanja über seinem Geburtstag zu informieren. 
spontaneous production of routines was fairly rare, parents' attempts to elicit them from their children were not: in general, parents tended to prompt the child to say the appropriate thing" (Blank/Berko 1980: 161).

Die Feinanalysen der einzelnen Grusssituationen bildeten die Grundlage für den Vergleich anhand des Kriterienkataloges, welcher in den Tabellen im nächsten Kapitel ersichtlich ist, und für die Interpretation.

\section{$6 \quad$ Auswertung}

Die folgenden Tabellen und Diagramme gewähren einen Überblick über das ausgewertete Datenmaterial und geben Anhaltspunkte für das Erstellen von Sequenzmustern im kindlichen Grussverhalten. Die Jahrgänge, nach denen die Kinder in den Tabellen 1 und 3 geordnet sind, sollen Aufschluss geben über einen möglichen Einfluss des Alters auf das Grussverhalten und somit auf die unterschiedlichen Stadien in der Sozialisation. Die Tabellen erfassen die am häufigsten beobachteten verbalen und nonverbalen Elemente des Grussverhaltens der Probanden, wobei ' $x$ ' eine einmalige Handlung bezeichnet. Für das Verbale sind die Adjacency Pairs 'Gruss und Gegengruss' sowie 'Befindlichkeitsfrage und Antwort' zentral. Sofern im Grussakt vom Gegenüber der Kinder keine Befindlichkeitsfrage geäussert wird, ist dies in der Tabelle in der Kategorie "Beantwortung Befindlichkeitsfrage" mit einem Schrägstrich markiert. Beim Nonverbalen sind sowohl allein auftretende wie auch sprachbegleitende Gesten aus allen vier Stadien des Salutations-Schemas nach Kendon (cf. Kendon 1990) aufgeführt. Die Kategorie "Verlegenheitsgeste" erfasst unter anderem die in der 'approach phase' auftretenden Phänomene 'body cross' und 'grooming'. Wenn Kinder mehrmals in Grusshandlungen eingebunden sind, werden sie für jede Grusshandlung erneut aufgeführt.

Die Diagramme veranschaulichen die Häufigkeit des Auftretens der verschiedenen Grusselemente im Grussverhalten der Kinder. Sie ist in normalisierter Form dargestellt. 100 Prozent entspricht der Anzahl aufgezeichneter Grüsse in der jeweiligen Kategorie. Tabelle 4 bezieht sich ausschliesslich auf die geäusserten Grussfloskeln. 


\subsection{Begrüssungen zwischen Erwachsenen und Kindern}

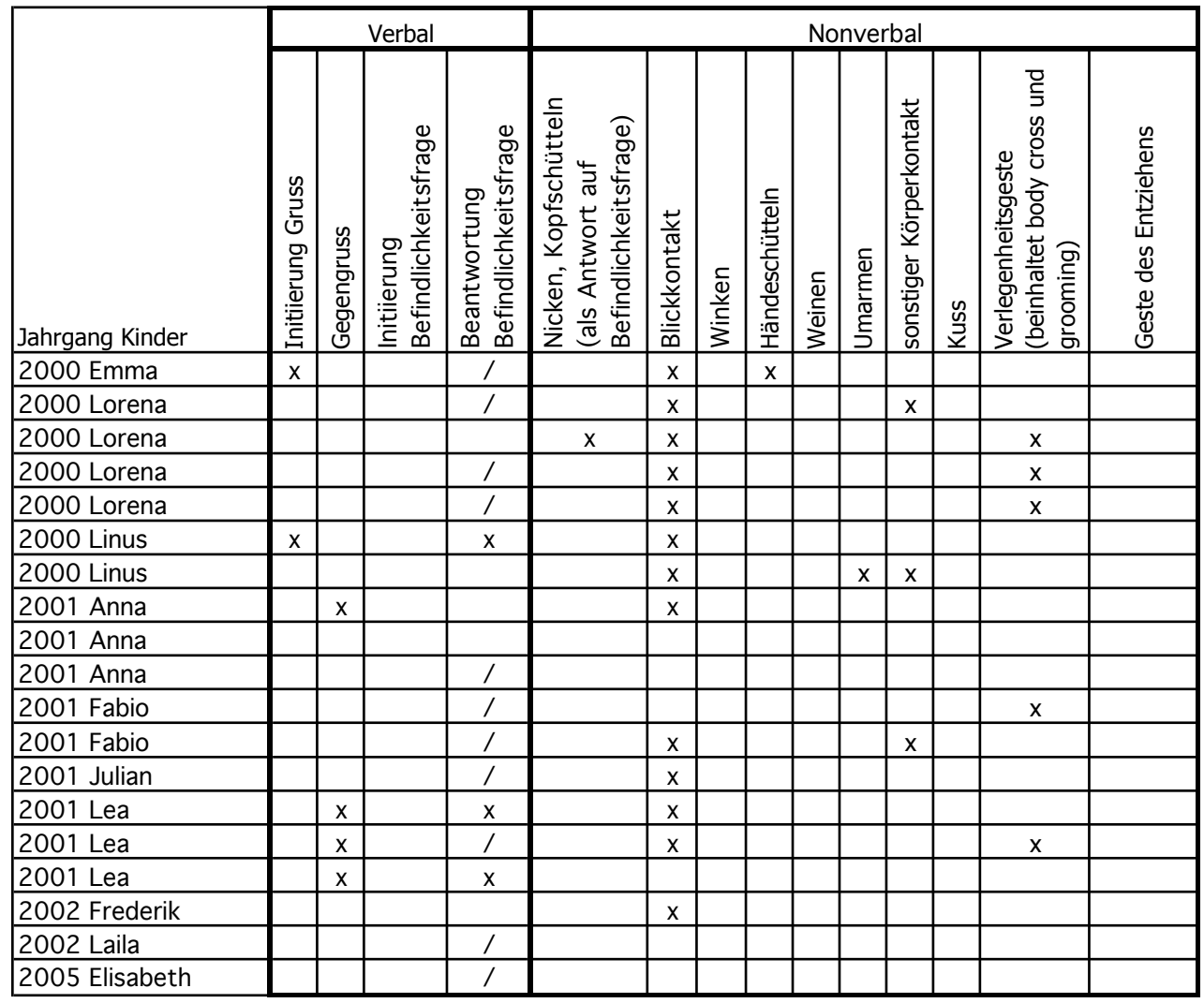

Tabelle 1: Begrüssungen zwischen Erwachsenen und Kindern

Die Erwachsenen, die an den in Tabelle 1 dargestellten Begrüssungen teilnehmen, sind entweder Mitarbeiterinnen des Kindergartens oder Untersuchungsleiterinnen. Mit Ausnahme zweier Fälle, in welche die ältesten Kinder der Gruppe involviert sind, werden alle Begrüssungen von den Erwachsenen initiiert. Nur zwei Kinder führen einen verbalen Gegengruss aus. In 13 Situationen wird der Gruss des Erwachsenen nicht verbal erwidert dies auch von Kindern, die in anderen Fällen verbal an einer Grusshandlung beteiligt waren. Befindlichkeitsfragen werden in vier von acht Fällen von den Kindern beantwortet, jedoch nie von ihnen gestellt.

In vier Situationen ist keinerlei Reaktion der Kinder auf den Gruss der Erwachsenen ersichtlich, weder verbal noch nonverbal. Die Kinder vermeiden hier sogar Blickkontakt. In insgesamt sechs der 19 Fälle stellt das begrüsste Kind keinen Blickkontakt her; einmal aber erfolgt trotzdem ein Gegengruss und die Beantwortung der Befindlichkeitsfrage. Zweimal wird auf eine Begrüssung nur mit einem Blick reagiert, wobei in einer Begrüssung zusätzlich eine Befindlichkeitsfrage an das Kind gerichtet wird. In drei Fällen kommt zum Blickkontakt eine Verlegenheitsgeste des Kindes hinzu. Ein Kind reagiert auf die Begrüssung neben Verlegenheitsgeste und Blickkontakt mit einem Gegengruss, ein anderes statt Gegengruss mit der Beantwortung der Befindlichkeitsfrage durch Nicken.

Der Körperkontakt respektive die Umarmung, welche in allen vier Fällen von den Kindern initiiert wird, richtet sich ausschliesslich an die Kindergärtnerin. 


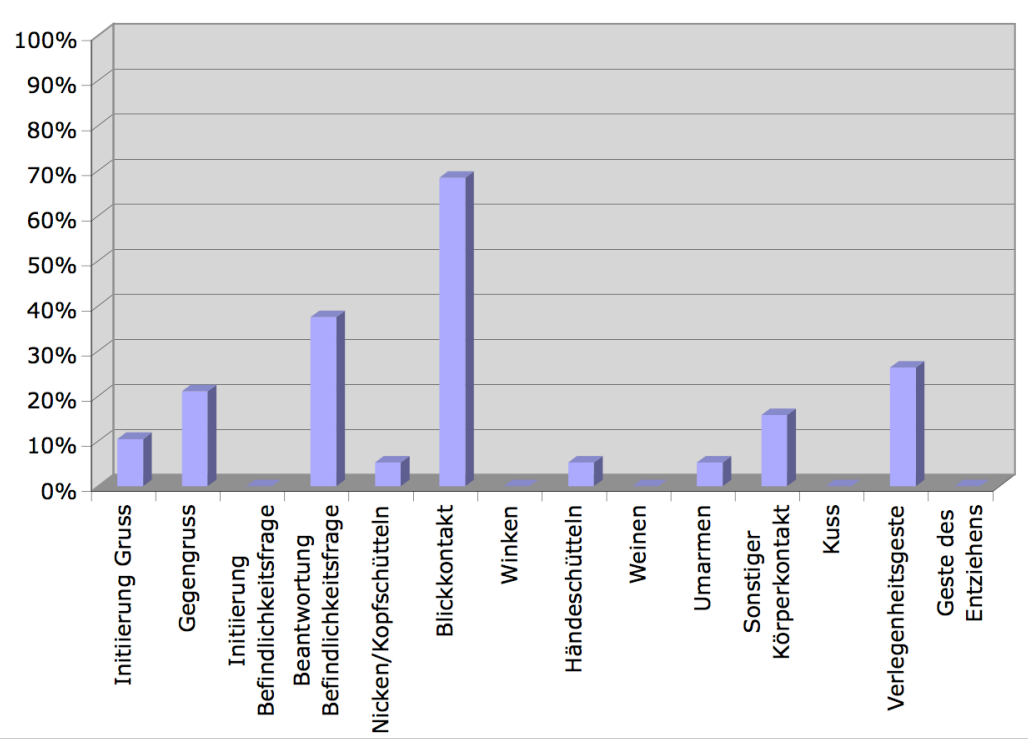

Diagramm 1: Begrüssungen zwischen Erwachsenen und Kindern n $=19$ Grusssituationen

\subsection{Begrüssungen unter Kindern}

Hier sind alle am Gruss beteiligten Kinder einzeln aufgeführt, wobei die Agierenden und die Reagierenden der gleichen Begrüssung in der Tabelle jeweils untereinander erscheinen.

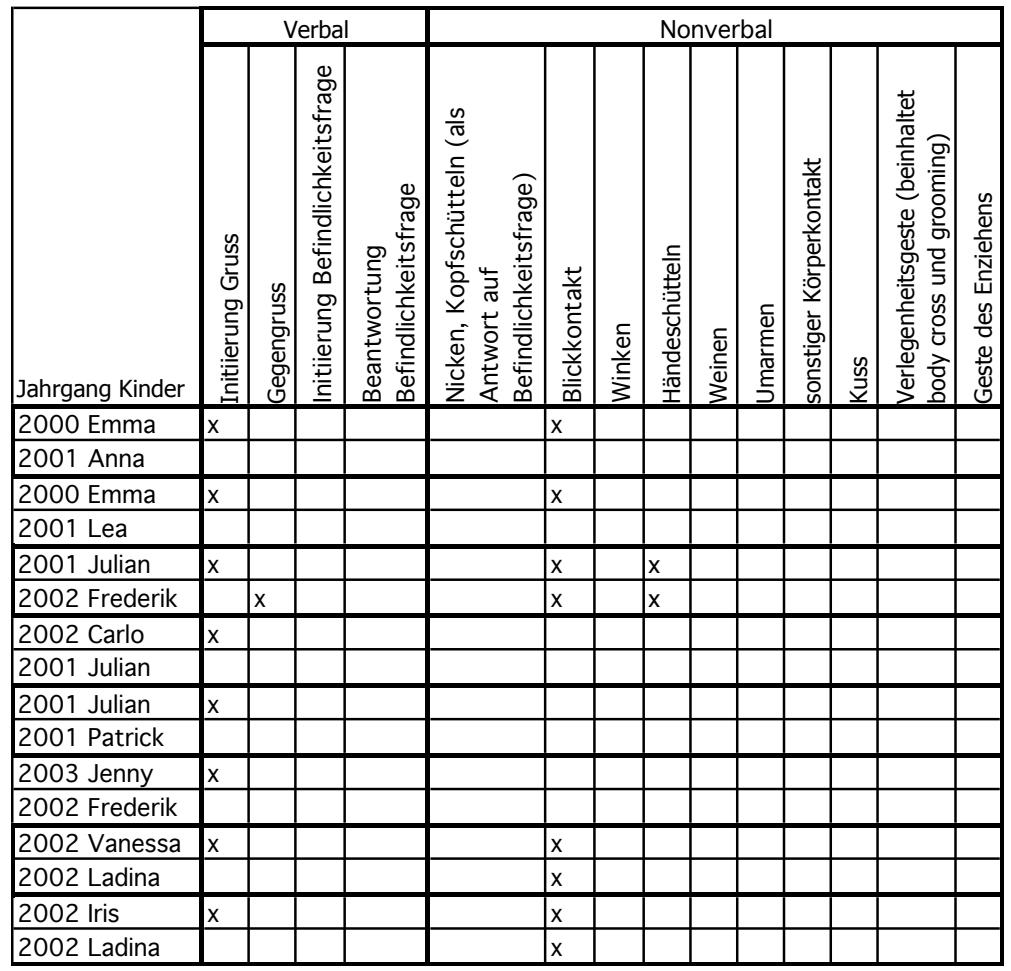

Tabelle 2: Begrüssungen unter Kindern

Nur eine der acht initiierten Begrüssungen wird verbal beantwortet. Neben der Grussfloskel beinhaltet diese auch Blickkontakt und Händeschütteln. In der Begrüssung unter Kindern werden keine Befindlichkeitsfragen geäussert. In sechs von acht Situationen wird der Gruss von einem älteren oder gleichaltrigen Kind initiiert; nur zweimal grüsst ein jüngeres Kind zuerst. 


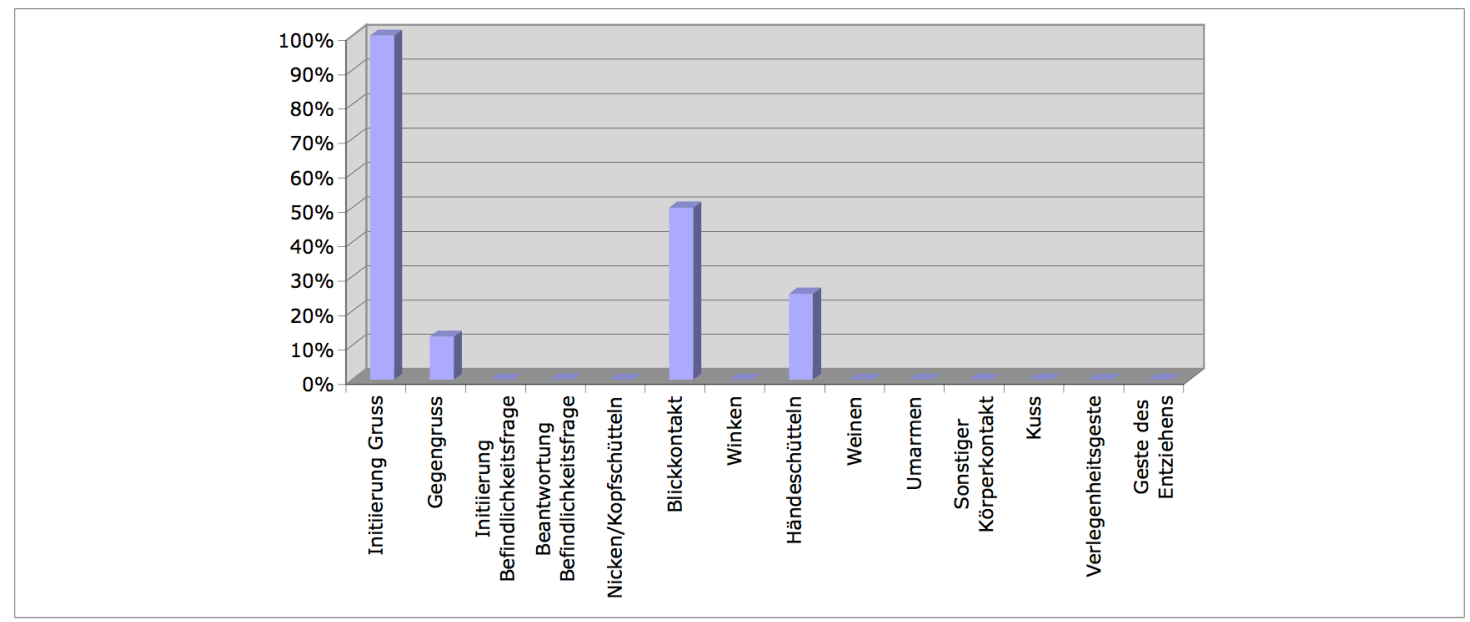

Diagramm 2: Begrüssungen unter Kindern $\mathrm{n}=\mathbf{8}$ Grusssituationen

\subsection{Verabschiedungen zwischen Erwachsenen und Kindern}

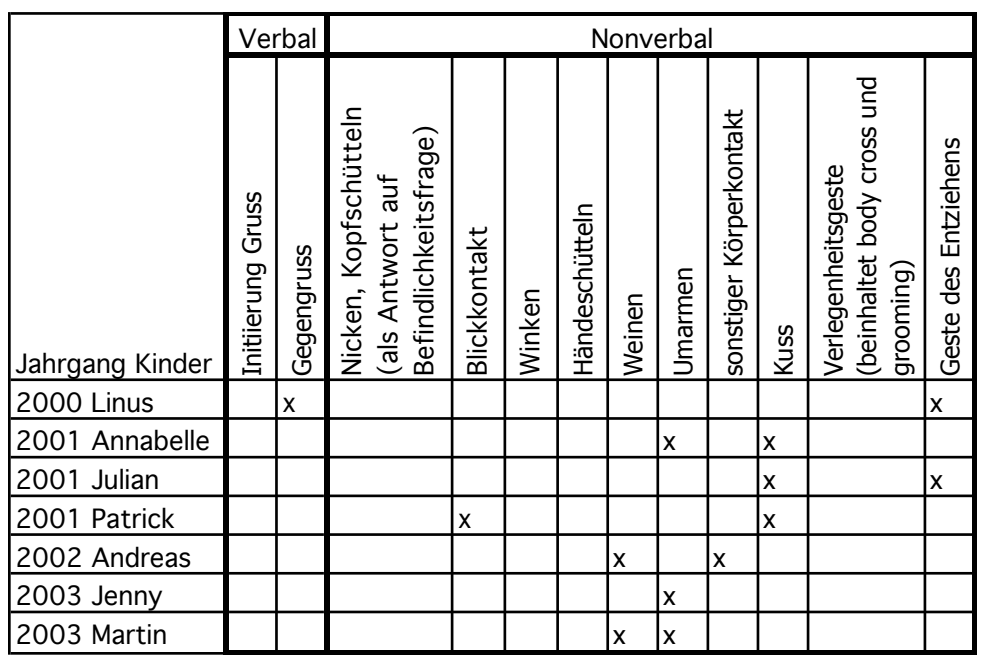

Tabelle 3: Verabschiedungen zwischen Erwachsenen und Kindern

Jede der aufgezeichneten Verabschiedungen enthält mindestens ein Element eines Verabschiedungsrituals von Seiten der Kinder. Nur ein Kind (und zwar das älteste der Gruppe) reagiert verbal auf die Verabschiedung der Mutter. Ebenfalls in nur einem Fall erwidert ein Kind im Moment des Abschieds den Blick des Elternteils. Zwei Kinder weinen stark beim Abschied; beide gehören zu den jüngeren Kindern des Kindergartens. Drei Kinder werden von ihren Eltern mit einem Kuss auf den Mund verabschiedet. In drei Fällen umarmen sich Eltern und Kindern für längere Zeit; einmal findet eine Berührung statt, welche keine eindeutige Umarmung ist. Die beiden ältesten Jungen führen die Verabschiedung zwar aus, aber nur mit einer Geste des Entziehens. 


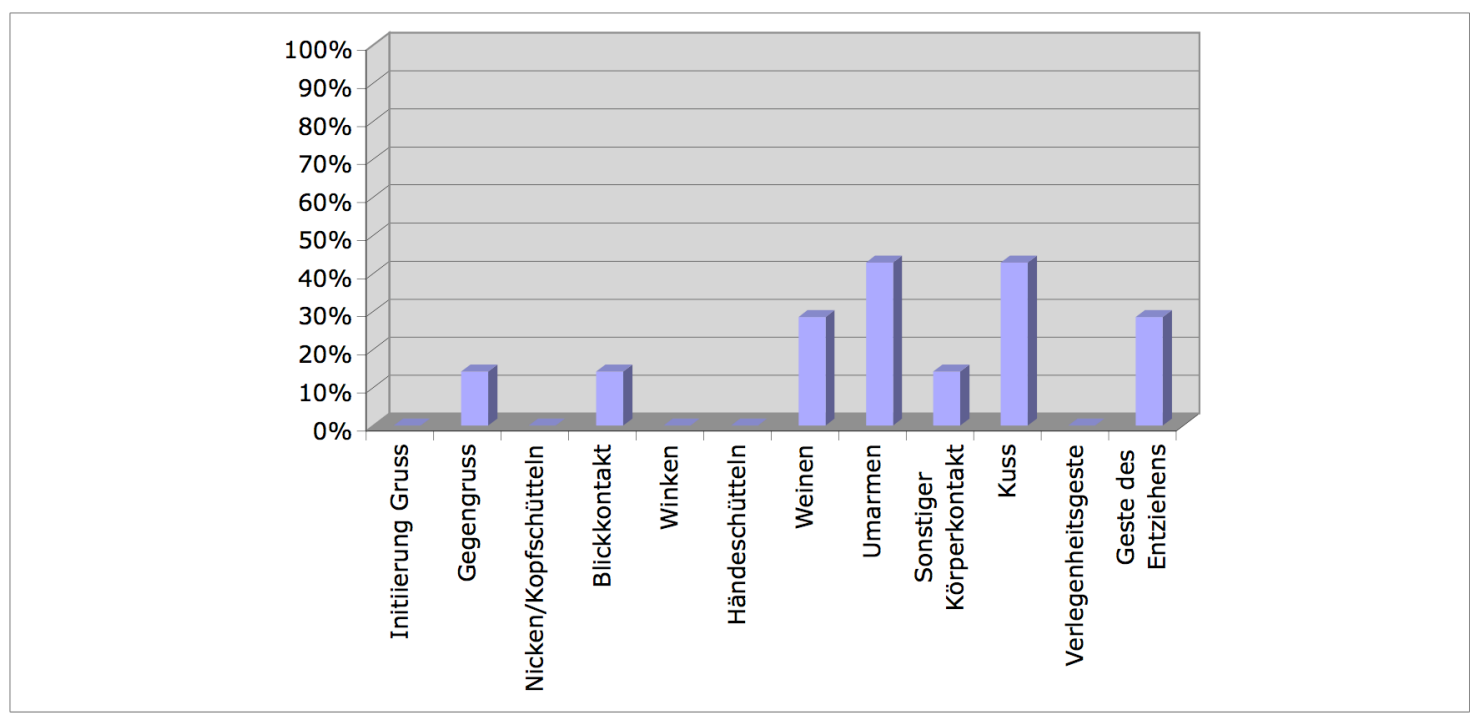

Diagramm 3: Verabschiedungen zwischen Erwachsenen und Kindern $n=7$ Grusssituationen

\subsection{Verwendete Grussfloskeln}

In der folgenden Tabelle sind alle Varianten des verbalen Grusses, welche im Datenmaterial auftreten, mit ihrer Häufigkeit erfasst.

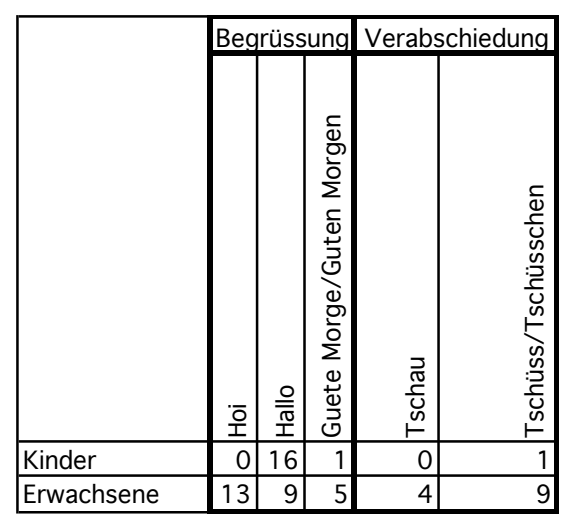

Tabelle 4: Repertoire der Grussfloskeln

Der Gruss "Hoi", welcher unter Erwachsenen die häufigste Begrüssungsfloskel ist, wird von Kindern nie benutzt, "Hallo" dagegen häufiger als von Erwachsenen. Der sprachlich aufwendigere Gruss "Guten Morgen" tritt bei beiden Gruppen auf. Bei Kindern ist nur eine Verabschiedungsfloskel aufgezeichnet, nämlich "Tschüss", welche auch unter Erwachsenen der beliebteste verbale Abschiedsgruss ist.

\section{$7 \quad$ Tendenzen und Fazit}

\subsection{Tendenzen}

Allgemein fällt auf, dass wenige an die Kinder gerichtete Grüsse von diesen mit einem Gegengruss erwidert werden. Während die Kinder in weniger als $20 \%$ der Fälle verbal zurückgrüssen, werden die Befindlichkeitsfragen mit einem Anteil von 50\% deutlich häufiger beantwortet. Der Blickkontakt wird in der Begrüssung erstaunlich wenig vermieden, obwohl scheinbar keine Reaktion auf die Begrüssung erfolgt. In der Verabschiedung scheint das 
Gegenteil der Fall zu sein; Blickkontakt tritt hier nur selten auf, obwohl jedes Kind in irgendeiner Form an der Verabschiedung teilnimmt. Die nonverbalen Grusselemente dominieren in Grusssituationen unter Kindern ebenso wie in denjenigen zwischen Kindern und Erwachsenen. Auffällig ist jedoch, dass die Kinder in den Begrüssungen mit den Erzieherinnen erheblich mehr verbale Aktivität zeigen als in den Verabschiedungen von ihren Eltern. In den Verabschiedungen von den Eltern hat der Körperkontakt einen höheren Stellenwert.

Von den 20 Kindergartenkindern initiieren lediglich acht eine Begrüssung, obwohl es das erste Wiedersehen nach zwei Wochen Ferien ist. Hierbei ist bezeichnend, dass alle Kinder, die den Erwachsenen gegenüber einen Gruss initiieren, mit sieben Jahren zu den ältesten Kindern der Gruppe gehören. Obwohl die Grüsse der Erwachsenen häufig sehr prägnant geäussert werden, bleiben sie oft unerwidert. Auf Grüsse von Peers reagieren die Kinder ebenso wenig. Dies wäre in der Kommunikation unter Erwachsenen unvorstellbar und würde als Verletzung des Höflichkeitsgebots angesehen werden. Die Verlegenheitsgesten, welche häufig auftreten, könnten ein Indiz dafür sein, dass die Kinder die Grusssituation als unangenehm empfinden. ${ }^{12}$ Dementsprechend sind es die älteren Kinder, welche in dieser kommunikativ herausfordernden Situation so reagieren, wie es nach dem Sequenzmuster des Grusses unter Erwachsenen üblich ist.

Das Repertoire an Grussfloskeln ist auffallend klein. In den 34 erfassten Grusssituationen finden sich lediglich fünf verschiedene Varianten, von denen die Kinder nur drei gebrauchen. Dass sie ausschliesslich die Floskel "Hallo" verwenden, deutet auf eine Anpassung an das Hochdeutsche hin, welche vermutlich aufgrund der vielen deutschen Kinder im Kindergarten stattgefunden hat. Dies gilt allerdings nicht für die Betreuerinnen, welche aufgrund ihrer schweizerischen Abstammung das schweizerdeutsche "Hoi" bevorzugen. Da die Verabschiedungen vor allem von hochdeutschsprachigen Eltern ausgeführt werden, überwiegt bei den Abschiedsfloskeln der Erwachsenen das "Tschüss".

\subsection{Fazit}

Das Repertoire an verwendeten Grussfloskeln, welches nicht die erwartete Vielfalt aufweist, ist nicht typisch für den schweizerdeutschen Grussgebrauch. Der hohe Anteil deutscher Kinder und Eltern haben das Sprachregister des Kindergartens insofern beeinflusst, als die Grussfloskeln, welche auch in Deutschland gebräuchlich sind, den ausschliesslich schweizerdeutschen vorgezogen werden. Dies lässt vermuten, dass die Wahl der Grussfloskeln weitgehend durch die Peergroup geprägt wird und weniger durch Autoritäten wie beispielsweise die Kindergärtnerin. Weiter wird die Auswahl an Grussformen durch die allgemein gültige Du-Anrede, welche zwischen allen beteiligten Personen ein Verhältnis der Nähe schafft, modifiziert.

Die Tatsache, dass Kinder einen an sie gerichteten Gruss oft verbal nicht erwidern, ist sowohl in der Kommunikation unter Kindern wie auch zwischen Kindern und Erwachsenen zu beobachten. Da der Grussaustausch unter Erwachsenen klar als Paarsequenz empfunden wird und ein unerwiderter Gruss als unhöflich gilt, macht das Ausbleiben des Gegengrusses das kindliche Grussverhalten besonders interessant. Die Häufigkeit dieses Ausbleibens lässt darauf schliessen, dass für Kinder beim Grüssen ein anderes Sequenzmuster gilt als für Erwachsene. In der Interaktion zwischen Erwachsenen wird die Phase erhöhter Zugänglichkeit üblicherweise in gegenseitigem Einverständnis, signalisiert durch den Grussaustausch, eingeleitet. Kinder scheinen den an sie gerichteten Gruss nicht nur als Angebot, sondern bereits als Einleitung der Phase erhöhter Zugänglichkeit zu betrachten, was den Gegengruss

${ }^{12}$ Dies ist auch bei Erwachsenen der Fall, da der Gruss immer ein "face-threatening act" ist (cf. Brown/Levinson 1987). 
überflüssig macht. Der Gruss, den das Gegenüber äussert, versichert dem Kind bereits die Aufmerksamkeit des Grüssenden. Die Kinder verweigern durch den ausbleibenden Gegengruss dem Grüssenden nicht die Zugänglichkeit: Dass sie für eine Interaktion bereit sind, lässt sich aus dem Blickkontakt, den sie aufnehmen, und dem Beantworten von Befindlichkeitsfragen schliessen. Beim Abschied, welcher die Phase erhöhter Zugänglichkeit beendet, verzichten die Kinder ebenfalls meist auf den verbalen Gegengruss. Auch hier scheint es ihnen zu genügen, die verbale Verabschiedung zu empfangen, ohne dem Gegenüber dieselbe zukommen zu lassen.

Dem nonverbalen Engagement und dem Körperkontakt hingegen kommt im kindlichen Sequenzmuster der Verabschiedung ein hoher Stellenwert zu. In Begrüssungen ist Körperkontakt seltener anzutreffen und der verbale Einsatz häufiger. Dies lässt sich mit dem Grad der Vertrautheit erklären, welcher zwischen Kindern und Betreuerinnen kleiner ist als zwischen Kindern und ihren Eltern. Der nonverbale Aufwand, der von Seiten der Kinder die Verabschiedung ausmacht, sowie deren Wiederholung zeigen die Wichtigkeit des Abschiedsrituals für Kinder. Somit ist die fehlende verbale Teilnahme am Abschied kein Zeichen der Abwertung desselben. Dies scheint den Erwachsenen bewusst zu sein, da sie nicht mit einer verbalen Grusserwiderung von Seiten der Kinder rechnen. Sie konstruieren den Gruss so, dass kaum Platz bleibt für einen Gegengruss der Kinder. Deshalb entsteht durch dessen Ausbleiben keine peinliche Pause. Die fehlende verbale Reaktion, welche in der Kommunikation unter Erwachsenen einen "face threat" (cf. Brown/Levinson 1987) darstellen würde ${ }^{13}$, wird hier akzeptiert.

Die Grusssituation wird nicht nur von Erwachsenen als kommunikativ herausforderndes Moment empfunden, sondern scheint auch bei Kindern Unbehagen auszulösen. Da Kinder dies (noch) nicht überspielen, kommt das Unbehagen häufig in Verlegenheitsgesten zum Vorschein. In Form von 'grooming' und 'body cross' treten solche auch in der 'approach phase' von Grusssituationen unter Erwachsenen zu Tage. In diesem Punkt deckt sich das kindliche Sequenzmuster mit demjenigen der Erwachsenen, was darauf hindeutet, dass auch Kinder Grusssituationen als potentielle "face threatening acts" empfinden. Gerade wegen der kommunikativ bedrohlichen Situation, die ein Gruss hervorruft, bemühen sich Erwachsene im Grussakt um gleichen Aufwand. Man versucht, sich dem Engagement des Gegenübers anzupassen, was durch die Befolgung des üblichen Sequenzmusters gewährleistet ist. Kinder hingegen kommen aufgrund ihres Sequenzmusters diesem Anspruch nach gleichem Aufwand nicht nach. Dies wird von Erwachsenen auch nicht erwartet, solange sich das Kind in der von Oksaar als "sprachliche Ontogenese" bezeichneten Phase befindet. In diesem Entwicklungsstadium werden Verstösse des Kindes gegen das Höflichkeitsgebot, welches einen Gegengruss fordert, nicht als Affront empfunden. Der Grossteil der Probanden des untersuchten Kindergartens befindet sich im Stadium der Ontogenese und agiert daher dem kindlichen Sequenzmuster gemäss. Bei einigen Kindern lässt sich feststellen, dass sie die wesentliche Phase des Spracherwerbs abgeschlossen haben. Diese haben das Sequenzmuster der Erwachsenen übernommen und wenden es, zumindest teilweise, an. Da es sich bei diesen Kindern um die ältesten der Gruppe handelt, kann daraus geschlossen werden, dass sie sich bereits in der "primären Erweiterungsstufe" befinden:

Sprachlich ist diese Stufe gekennzeichnet durch die verfeinerte Verwendung lexikalischer und grammatischer Elemente - originale Wortbildungen, steigende Verwendung von hypotaktischen Konstruktionen [...]. Sie ist ferner gekennzeichnet durch einen sich ausbreitenden Sozialisierungsprozess, durch zunehmende Umwelteinflüsse, da der Kontakt mit Menschen ausserhalb der Familie durch Spielgefährten und/oder Kindergarten zunimmt (Oksaar 1987: 12).

\footnotetext{
13 Im Gruss als Einstieg in eine Kommunikation etablieren die Beteiligten ihre Beziehung, indem sie ihr "face" exponieren, wobei immer mit einem allfälligen Angriff auf dasselbe gerechnet werden muss.
} 
In diesem Sozialisierungsprozess haben die älteren Kinder gelernt, dass man "manchmal reden muss, obwohl man nicht will" (Klein 1989: 10), so zum Beispiel in Begrüssungen und Verabschiedungen. Sie scheinen jedoch das kindliche Sequenzmuster nicht gänzlich abgelegt zu haben, da sie in gewissen Situationen, vor allem in der Kommunikation mit anderen Kindern, noch darin verharren.

Die Eltern scheinen zu erkennen, welche Bedeutung der Aneignung des üblichen Sequenzmusters - als Zeichen für die fortgeschrittene Sozialisation - zukommt. Deshalb versuchen vor allem die Eltern von Kindern, die nahe am Übergang zur primären Erweiterungsstufe stehen, diesen Lernprozess durch Ermahnung zu korrektem Grüssen zu unterstützen.

The enormous effort that parents put into teaching routines and the far-reaching consequences of failure to use them belie the notion that they are unimportant or somehow incidental to language acquisition. While a child might get by in the world without ever using the passive, or even a common lexical item like ball, the child who does not learn to say hi, thanks, and goodbye will become a despised member of society (Blank/Berko 1980: 166).

Eine wiederholte Datenaufnahme wäre interessant, um die hier festgehaltenen Tendenzen zu bestätigen und die Entwicklung der einzelnen Kinder nachzuvollziehen. Da dem Gruss als kommunikative Praktik in unserer Gesellschaft ein grosser Stellenwert zukommt, wäre eine eingehende Untersuchung des Themas von Nöten, um auch den Verlauf der Grusskompetenz über das Vorschulalter hinaus zu verfolgen.

Grüssen ist eine kommunikative Praktik, welche erlernt werden muss. Die vorliegende Pilotstudie zeigt den Zusammenhang zwischen dem Alter der Kinder - vier bis sieben Jahre und dem Beherrschen der konventionellen Grusspraktik. In diesen vier Jahren findet also ein Lernprozess statt, welcher einen erheblichen Teil der Sozialisation ausmacht: Die Kinder werden von Kleinkindern, welche sich noch nicht an soziale Regeln halten müssen, zu Schulkindern, welche die Gebote der Gesellschaft zu befolgen haben, um Teil derselben zu werden.

\section{Bibliographie}

Abraham, Werner (1988): Terminologie zur neueren Linguistik. 2., völlig neu bearb. und erw. Aufl. Tübingen.

Blank Greif, Esther/Berko Gleason, Jean (1980): "Hi, thanks, and goodbye: More routine information". In: Language in Society 9/1: 159-166.

Brown, Penelope/Levinson, Stephen C. (1987): Politeness. Some universals in language usage. . Cambridge. (= Studies in Interactional Sociolinguistics 4).

Burger, Harald et al. (1989): "Richtlinien zur Transkription von Texten". http://www.ds.uhz.ch/Linguistik/. Stand 10.4.2008.

Deppermann, Arnulf (1999): "Gespräche analysieren. Eine Einführung in konversationsanalytische Methoden". In: Bohnsack, Ralf et al. (ed.): Qualitative Sozialforschung. Opladen: $39-48$.

Field, Tiffany et al. (1984): "Leave-takings and Reunions of Infants, Toddlers, Preschoolers, and Their Parents". In: Child Development 55/1: 628-635.

Glück, Helmut (Hrsg.) (2005): Metzler Lexikon Sprache. Stuttgart.

Goffman, Erving (1982): Das Individuum im öffentlichen Austausch. Mikrostudien zur öffentlichen Ordnung. Frankfurt am Main.

Hartmann, Dietrich (1973): "Begrüssungen und Begrüssungsrituale. Überlegungen zu Verwendungsweisen sprachlicher Symbolik in kommunikativen Handlungsmustern". ZGL 1/2: $133-162$.

Kendon, Adam (1990): Conducting interaction. Patterns of behavior in focused encounters. Cambridge.

Klein, Wolfgang (1989): "Sprechen lernen - das Selbstverständlichste von der Welt". Zeitschrift für Literaturwissenschaft und Linguistik 73: 7-17. 
Kohrt, Manfred (1984): "Von hallo! wie geht's bis tschüss! mach's gut! - Grüsse, Grussformeln und Verwandtes im gegenwärtigen deutschen Sprachgebrauch". In: Kürschner Wilfried et al. (ed.): Sprachtheorie, Pragmatik, Interdisziplinäres. Akten des 19. Linguistischen Kolloquiums. Tübingen: 173-184.

Linke, Angelika et al. (2004): Studienbuch Linguistik. 5., erweiterte Auflage. Tübingen. (= Reihe Germanistische Linguistik 121).

Montessori, Renilde (1983): Uns drückt keine Schulbank. Montessori-Erziehung im Bild. Stuttgart.

Oksaar, Els (1987): Spracherwerb im Vorschulalter. Einführung in die Pädolinguistik. 2., erw. Aufl. Stuttgart. (= Sprache und Literatur 124).

Schegloff, Emanuel A. (1972): "Sequencing in Conversational Openings". In: Gumperz, John J./Hymes, Dell (ed.): Directions in Sociolinguistics. The Ethnography of Communication. New York et al.: 346-380.

Selting, Margret et al. (1998): "Gesprächsanalytisches Transkriptionssystem (GAT)". Linguistische Berichte 173: 91-122

Zakharine, Dmitri (2005): Von Angesicht zu Angesicht. Der Wandel direkter Kommunikation in der ost- und westeuropäischen Neuzeit. Konstanz. 


\section{Anhang}

Das gesprächsanalytische Transkriptionssystem GAT nach Selting et al. (1998): 114-115.

Basistranskript:

Sequenzielle Struktur/Verlaufsstruktur

[ ]

[ ]

$=$

Überlappungen und Simultansprechen

schneller, unmittelbarer Anschluss neuer Turns oder Einheiten

\section{Pausen}

(.)

$(-),(--),(--)$
Mikropause

kurze, mittlere, längere Pausen von ca. 0.25-0.75 Sek.; bis ca. 1 Sek. geschätzte Pause, bei mehr als ca. 1 Sek. Dauer gemessene Pause (Angabe mit zwei Stellen hinter dem Punkt)

Sonstige segmentale Konventionen

und $=$ äh

$:,::,:::$

äh, öh, etc.

\section{Lachen}

so(h)o

haha hehe hihi

((lacht))

Rezeptionssignale

$\mathrm{hm}$, ja, nein, nee

$\mathrm{h}=\mathrm{hm}, \mathrm{ja}=\mathrm{a}$, nei=ein,

nee $=\mathrm{e}$

'hm'hm

Akzentuierung

akZENT

ak!ZENT!
Verschleifungen innerhalb von Einheiten

Dehnung, Längung, je nach Dauer

Verzögerungssignale, sog. "gefüllte Pausen"

Abbruch durch Glottalverschluss

Lachpartikeln beim Reden

silbisches Lachen

Beschreibung des Lachens

einsilbige Signale

zweisilbige Signale

mit Glottalverschlüssen, meistens verneinend

\section{Tonhöhenbewegung am Einheitenende}

?

,

$-$

;
Primär- bzw. Hauptakzent

extra starker Akzent

hoch steigend

mittel steigend

gleich bleibend

mittel fallend

tief fallend 
Sonstige Konventionen

((hustet))

$<<$ hustend $>\quad>$

$<<$ erstaunt $>\quad>$

$(\quad)$

(solche)

al(s)o

(solche/welche)

$((\ldots))$

$\rightarrow$ para- und aussersprachliche Handlungen u. Ereignisse

sprachbegleitende para- und aussersprachliche Handlungen

und Ereignisse mit Reichweite

interpretierende Kommentare mit Reichweite

unverständliche Passage je nach Länge

vermuteter Wortlaut

vermuteter Laut oder Silbe

mögliche Alternativen

Auslassung im Transkript

Verweis auf im Text diskutierte Transkriptzeile 